\title{
Influência dos sistemas de controle gerencial e da criatividade sobre o desempenho no trabalho
}

\author{
ITZHAK DAVID SIMÃo KAVESKI ${ }^{1}$ \\ ILSE MARIA BEUREN ${ }^{2}$ \\ ${ }^{1}$ Universidade Federal de Mato Grosso do Sul (UFMS), Corumbá - MS, Brasil \\ ${ }^{2}$ UnIVERSIDAdE FEDERAL DE SANTA CATARINA (UFSC), FLORIANÓPOLIS - SC, BRASIL
}

\begin{abstract}
Resumo
Este estudo analisa a influência dos sistemas de controle gerencial (SCG) sobre o desempenho no trabalho, mediada pela criatividade individual. Foram consideradas as quatro alavancas de controle do modelo de Simons (1995): sistemas de crenças, sistemas de limites, sistemas de controle interativo e sistemas de controle diagnóstico. Uma pesquisa de levantamento foi realizada com empresas industriais consideradas inovadoras no Brasil, e obteve-se um retorno de 126 respostas válidas. Os resultados da modelagem de equações estruturais indicaram que há relações significativamente diferentes entre os SCG e a criatividade individual: algumas influenciam positivamente os sistemas de crenças e os sistemas de controle interativo; enquanto outras influenciam negativamente os sistemas de controle diagnóstico. Averiguou-se ainda que os sistemas de crenças influenciam de forma positiva o desempenho no trabalho, mediados de forma parcial pela criatividade individual. Estes resultados denotam a importância dos SCG para estimular a criatividade individual dos gestores, para auxiliar na realização das tarefas na organização e, consequentemente, aumentar o desempenho no trabalho. Conclui-se que os sistemas de crenças, os sistemas de controle interativo e os sistemas de controle diagnóstico influenciam na criatividade individual, tal como a criatividade influencia no desempenho no trabalho. Por fim, considera-se que a não significância da relação entre sistemas de limites e criatividade individual instiga novas pesquisas.
\end{abstract}

Palavras-chave: Sistemas de controle gerencial. Desempenho no trabalho. Criatividade.

\section{Influence of management control systems and creativity on job performance}

\begin{abstract}
This study analyzes the influence of managerial control systems (MCS) on job performance, mediated by individual creativity. Simons's (1995) four levers of control were considered: belief systems, boundary systems, interactive control systems, and diagnostic control systems. A survey was conducted with industrial companies considered to be innovative in Brazil, obtaining 126 valid responses. The results of the structural equation modeling indicated a significantly different relationship between MCS and individual creativity. Some positively influence belief systems and interactive control systems, while others negatively influence diagnostic control systems. It was also found that belief systems positively influence job performance, partly mediated by individual creativity. These results denote the importance of McS to stimulate managers' individual creativity, to assist in tasks carried out in the organization and, to increase job performance. The study concludes that belief systems, interactive control systems, and diagnostic control systems influence individual creativity, just as creativity influences job performance. In addition, the non-significance of the relationship between boundary systems and individual creativity deserves further research.
\end{abstract}

Keywords: Management control systems. Job performance. Creativity.

\section{Influencia de los sistemas de control de gestión y creatividad sobre el desempeño en el trabajo}

\section{Resumen}

Este estudio analiza la influencia de los sistemas de control de gestión (SCG)) sobre el desempeño en el trabajo, mediada por la creatividad individual. Se consideraron las cuatro palancas de control del modelo de Simons (1995): sistemas de creencias, sistemas de límites, sistemas de control interactivo y sistemas de control de diagnóstico. Se realizó una encuesta con empresas industriales consideradas innovadoras en Brasil, y se obtuvieron 126 respuestas válidas. Los resultados del modelado de ecuaciones estructurales indicaron que hay relaciones significativamente diferentes entre SCG y la creatividad individual: algunas influyen positivamente en los sistemas de creencias y los sistemas de control interactivo; mientras que otras influyen negativamente en los sistemas de control diagnóstico. También se averiguó que los sistemas de creencias influyen positivamente en el desempeño en el trabajo, mediados de forma parcial por la creatividad individual. Estos resultados denotan la importancia de los SCG para estimular la creatividad individual de los gestores, para auxiliar en la realización de las tareas en la organización y, consecuentemente, aumentar el desempeño en el trabajo. Se concluye que los sistemas de creencias, sistemas de control interactivo y sistemas de control diagnóstico influencian en la creatividad individual, tal como la creatividad influye en el desempeño del trabajo. Por fin, se considera que la no significancia de la relación entre sistemas de límites y creatividad individual instiga nuevas investigaciones.

Palabras clave: Sistemas de control de gestión. Desempeño en el trabajo. Creatividad. 


\section{INTRODUÇÃO}

Os sistemas de controle gerencial (SCG) incorporam todos os sistemas usados pelos gestores para assegurar a congruência das decisões e do comportamento dos subordinados com as estratégias e os objetivos organizacionais (MERCHANT e VAN DER STEDE, 2017). São concebidos em um formato para que os empregados aceitem os objetivos da organização, conforme os padrões e planos pré-estabelecidos, e, ao mesmo tempo, sejam obrigados a sacrificar os seus interesses individuais para alcançar o que foi pré-estabelecido pela empresa (ADLER e CHEN, 2011). Nesse sentido, diversas pesquisas têm se concentrado em compreender de que forma os SCG influenciam o desempenho individual e/ou organizacional (HALL, 2011).

Algumas pesquisas relacionaram os SCG com o desempenho no trabalho, por exemplo, de Burney e Widener (2007), Hall $(2008$, 2011), Marginson, McAulay, Roush et al. (2014). Estes estudos destacam o papel de mecanismos cognitivos (clareza de papéis) e motivacionais (empowerment psicológico) para entender como os SCG afetam o desempenho individual. Observa-se uma lacuna quanto ao exame dos efeitos diretos e/ou indiretos dos componentes dos SCG sobre o desempenho no trabalho, todavia pode haver diferenças teórico-empíricas entre os modelos de efeitos diretos e indiretos (HALL, 2008).

Outro aspecto que tem recebido atenção neste contexto é a criatividade individual, identificada como essencial na capacidade da organização de inovar para se manter competitiva (AMABILE, 1983; DAVILA, FOSTER e OYON, 2009). Assim, os SCG podem ser utilizados ou para gerar ou para restringir a criatividade na organização (AMABILE, 1983; DAVILA, FOSTER e OYON, 2009). Ou seja, por um lado, a restrição da criatividade pelos SCG consiste em implantar uma padronização que restrinja a criatividade (MOULANG, 2015); por outro lado, o estímulo à criatividade pelos SCG consiste em alterar os padrões das atividades organizacionais, incentivando a criatividade e a procura de novas oportunidades (SIMONS, 1995).

Davila, Foster e Oyon (2009) destacam que em ambientes de trabalho fortemente influenciados pelos SCG, a criatividade individual pode ser estimulada. Todavia defendem a necessidade de mais pesquisas sobre a criatividade individual. De acordo com Gong, Huang e Farh (2009), a criatividade individual pode ser benéfica para os resultados no trabalho, o que é suscetível de beneficiar as organizações. Porém, Gilson (2008) alerta que essa criatividade não poderá impactar negativamente o desempenho do indivíduo no trabalho. $\mathrm{O}$ autor argumenta, no entanto, que há necessidade de mais estudos empíricos para averiguar se a criatividade individual resulta em melhor desempenho individual no ambiente organizacional.

Com base no exposto, a questão que orienta este estudo é a seguinte: qual a influência dos sistemas de controle gerencial (sistemas de crenças, sistemas de limites, sistemas de controle interativo e sistemas de controle diagnóstico) sobre o desempenho no trabalho (desempenho individual do gestor na empresa), mediada pela criatividade individual (envolvimento do gestor com atividades criativas na empresa)? Desse modo, o objetivo do presente estudo é verificar a influência dos sistemas de controle gerencial sobre o desempenho no trabalho, mediada pela criatividade individual.

Pesquisas anteriores, como de Webster (2006) e Moulang (2015), consideraram somente os sistemas de controle interativo, enquanto outras pesquisas, como de Speklé, Van Elten e Widener (2017), consideraram as quatro alavancas de Simons de forma conjunta. Além disto, estas pesquisas utilizaram o empowerment psicológico como variável mediadora entre criatividade individual e SCG. O presente estudo contribui para o quadro teórico do problema analisando separadamente as quatro alavancas de Simons e considerando a criatividade individual como mediadora entre os SCG e o desempenho no trabalho.

Este estudo se justifica por investigar os antecedentes e consequentes da criatividade individual, ou seja, o que leva um indivíduo a ser criativo para beneficiar as suas tarefas e a organização como um todo (ZHOU e SHALLEY, 2008; GONG, HUANG e FARH, 2009). Portanto, apresenta uma perspectiva diferenciada da relação entre os SCG e o desempenho no trabalho, utilizando a criatividade individual como variável mediadora. Também se diferencia de pesquisas que examinaram como os mecanismos cognitivos e motivacionais explicam as relações entre os SCG e o desempenho no trabalho (BURNEY e WIDENER, 2007; HALL, 2008, 2011; MARGINSON, MCAULAY, ROUSH et al., 2014), ao verificar como os SCG podem melhorar o desempenho individual dos gestores pela sua criatividade.

Justifica-se ainda o estudo por considerar os SCG, com as alavancas de controle (Levers of Control - LOC's) do modelo de Simons (1995), como antecedentes da criatividade individual. Neste estudo, optou-se por utilizar o modelo de Simons (1995), pois, para lidar com a tensão entre a necessidade de alcançar os objetivos estabelecidos e expandir a busca por novas oportunidades, inovação e iniciativas estratégicas, os gestores utilizam as quatro alavancas de controle, as quais criam tensões dinâmicas e garantem o controle da organização. 


\section{REFERENCIAL TEÓRICO E HIPÓTESES DA PESQUISA}

A literatura aponta os SCG como antecedentes da criatividade e do desempenho, pois eles visam equilibrar as tensões dinâmicas decorrentes da necessidade organizacional em conceder liberdade para o desenvolvimento da criatividade dos indivíduos e, ao mesmo tempo, assegurar o controle dos recursos, considerando ainda que estas relações emergem em meio a um ambiente de intensa competição (SIMONS, 1995). Assim, esta seção apresenta um referencial teórico sobre a relação entre os SCG, a criatividade e o desempenho, além de formular as três hipóteses da pesquisa.

\section{Sistemas de controle gerencial e criatividade}

Anthony (1965) cunhou o conceito de Sistema de Controle Gerencial (SCG) para descrever a totalidade de processos e instituições formais que permitem que a administração controle o uso de recursos, de tal forma que as metas e as estratégias organizacionais sejam, respectivamente, atendidas e implementadas. Para Simons (1995), os SCG consistem em rotinas e procedimentos baseados na informação que são usados pelos gestores para influenciar nas atividades organizacionais.

Simons (1995) propôs um modelo de SCG composto por quatro alavancas de controle (Levers of Control - LOC's): (i) sistemas de crenças, responsáveis por fornecer valores fundamentais da organização aos seus membros, tais como, missão, visão e valores; (ii) sistemas de limites, responsáveis por delinear um comportamento aceitável dos membros na organização; (iii) sistemas de controle interativo, responsáveis por promover e possibilitar o envolvimento face a face entre subordinados e pares; e (iv) sistemas de controle diagnóstico, responsáveis por acompanhar, monitorar e avaliar o desempenho dos membros. Simons (1995) postula que os SCG podem ser utilizados de forma conjunta pelos gestores.

Pesquisas como as de Webster (2006), Moulang (2015) e Speklé, Van Elten e Widener (2017) investigaram o SCG como um antecedente da criatividade nas organizações. Analisaram em que medida o SCG pode favorecer ou inibir a criatividade nas organizações. A criatividade é entendida como a geração de ideias novas e úteis por um indivíduo ou pequeno grupo de indivíduos (AMABILE, 1983). Ela se manifesta pelo desempenho criativo de um indivíduo na elaboração de ideias úteis relativas aos produtos, aos serviços, às tarefas, aos métodos ou aos procedimentos (ZHANG e BARTOL, 2010).

A criatividade é essencial para a capacidade de inovação de uma organização, podendo tanto ser benéfica ao desempenho no trabalho individual, quanto para o resultado global da organização (GEORGE e ZHOU, 2002; GONG, HUANG e FARH, 2009). Por sua vez, os SCG podem ser usados para aumentar ou restringir esta criatividade nas organizações (MOULANG, 2015), no sentido de conceder liberdade para o seu desenvolvimento pelos indivíduos e, ao mesmo tempo, assegurar o controle dos recursos.

Webster (2006) explorou a natureza das relações entre o uso interativo dos SCG e resultados da criatividade por meio do desempenho individual de gerentes de organizações australianas. Seu estudo evidenciou que o uso interativo dos SCG pode estimular sentimentos associados ao empowerment psicológico e, consequentemente, aumentar a criatividade e o desempenho individual. Ele concluiu que os SCG apresentam potencial para estimular a criatividade, mas reconhece a dificuldade de mensurar a criatividade, o que representa uma lacuna de pesquisa.

Moulang (2015) desenvolveu um arcabouço conceitual para relacionar o uso dos SCG à criatividade, incorporando o empowerment psicológico como variável mediadora. Os resultados mostram que o empowerment psicológico ajuda a explicar a relação entre o uso interativo dos SCG e a criatividade. O autor concluiu que o uso interativo dos SCG afeta os processos psicológicos dos indivíduos, suas cognições de empowerment psicológico, e que o empowerment psicológico afeta a criatividade individual, ao proporcionar respostas alternativas e potencialmente mais criativas no engajamento de tarefas.

Speklé, Van Elten e Widener (2017) desenvolveram um modelo teórico baseado nas LOC's de Simons (1995) para investigar o paradoxo entre criatividade individual e SCG, mediado pelo empowerment psicológico. Os resultados do estudo evidenciaram que as LOC's fornecem informações aos empregados acerca dos problemas da organização: elas mostram questões organizacionais que demandam ação criativa, atuam como agentes motivadores na tomada de decisões e fornecem aos empregados liberdade para serem criativos dentro de determinado limite. Desse modo, os autores argumentam que é relevante identificar a influência dos SCG sobre o empowerment psicológico e desse sobre a criatividade, já que tal cenário permeia o ambiente de tensão dinâmica, necessário à criatividade na presença de SCG.

Depreende-se do exposto que a diversidade de dimensões relacionadas com a criatividade no escopo organizacional pode ter influenciado nos resultados conflitantes das pesquisas. Assim, formula-se a primeira hipótese com o seguinte direcionamento:

- $\mathrm{H}_{1}$ : Há uma relação positiva entre os sistemas de controle gerencial e a criatividade individual. 
A confirmação da hipótese $\mathrm{H}_{1}$ indicará que os SCG figuram como elemento antecedente da criatividade individual, suscitando a criatividade individual dos gestores. Neste caso, os gestores podem usar os SCG para aumentar a criatividade individual nas organizações.

\section{Criatividade e Desempenho no Trabalho}

A criatividade constitui-se de expertise, pensamento criativo e motivação para a tarefa (AMABILLE, 1983). Expertise é o início de todo o trabalho criativo (AMABILLE, 1983) e refere-se ao conhecimento de determinado domínio, que é influenciado pela educação formal e informal do indivíduo, além de suas habilidades motoras, cognitivas e perceptivas (ZHOU e SHALLEY, 2008). O pensamento criativo agrega valor ao desempenho criativo e abre novas perspectivas para a resolução de problemas, pautado na persistência de evolução de algo já existente (ZHOU e SHALLEY, 2008). Motivação para a tarefa compreende a atitude individual no cumprimento de determinada tarefa e a percepção sobre a sua motivação para realizá-la (ZHOU e SHALLEY, 2008). Ela pode ser intrínseca, ao surgir da reação positiva de um indivíduo para a qualidade de realização de uma tarefa, ou extrínseca, quando a motivação procede de fontes externas à tarefa (AMABILLE, 1983; ZHOU e SHALLEY, 2008).

Em âmbito organizacional, estes aspectos influenciam o desempenho no trabalho. Empregados criativos tendem a ser mais hábeis na solução de problemas, o que se reflete em níveis superiores de desempenho (GONG, HUANG e FARH, 2009). Isso é corroborado pela pesquisa de Gong, Huang e Farh (2009), que investigaram a relação entre a criatividade dos empregados e o seu desempenho no trabalho, esse avaliado pelos supervisores e pelas vendas. Dentre os resultados, foi constatado que a criatividade dos empregados se relaciona positivamente com o desempenho no trabalho. Tais resultados denotam a importância da criatividade dos indivíduos nas organizações para melhorar o seu desempenho no trabalho, o desempenho da unidade de trabalho e/ou da organização como um todo.

A criatividade, entendida como um construto de desempenho criativo, foi relacionada com o desempenho no trabalho nas pesquisas de Zhang e Bartol (2010) e Bodla e Naeem (2014). Zhang e Bartol (2010) investigaram questões mais amplas das relações entre o engajamento no processo de trabalho criativo, o desempenho criativo e o desempenho no trabalho, e constataram uma mediação parcial na relação entre o desempenho criativo e o desempenho no trabalho. Bodla e Naeem (2014) desenvolveram um quadro teórico para verificar a influência da motivação intrínseca no desempenho de vendas utilizando o desempenho criativo como mediador parcial, e observaram que o desempenho criativo da força de vendas é alimentado pela motivação intrínseca.

Diante do exposto, em que a criatividade é abordada como preditora do desempenho no trabalho, enuncia-se a segunda hipótese da pesquisa:

- $\mathrm{H}_{2}$ : Há uma relação positiva entre a criatividade individual e o desempenho no trabalho.

A confirmação da hipótese $\mathrm{H}_{2}$ indicará que os gestores que buscam novas ideias na prestação de serviços, ou que primam pela melhoria nos processos e procedimentos das tarefas, conseguem melhorar a qualidade e a eficácia do serviço.

\section{Sistemas de controle gerencial e desempenho no trabalho}

Os efeitos dos SCG sobre o desempenho individual dos trabalhadores têm sido analisados em diferentes contextos. Os resultados das pesquisas revelam que os SCG influenciam indiretamente o desempenho dos gestores por meio de variáveis de mediação, como, ambiguidade de papéis (BURNEY e WIDENER, 2007; MARGINSON, MCAULAY, ROUSH et al., 2014), conflito de papéis e informações relevantes para o trabalho (BURNEY e WIDENER, 2007), empowerment psicológico (HALL, 2008; MARGINSON, MCAULAY, ROUSH et al., 2014), clareza de papéis (HALL, 2008), desenvolvimento de modelos mentais dos gestores (HALL, 2011).

Burney e Widener (2007) examinaram o efeito mediador do conflito de papéis, a ambiguidade de papéis e as informações relevantes para o trabalho na relação entre os SCG e o desempenho gerencial. O resultado foi baseado em dados colhidos entre mais de 700 membros do Institute of Management Accountants (IMA) com cargo de gerente. A pesquisa evidencia que a relação entre os SCG e o desempenho gerencial é mediada por informações relevantes para o trabalho, pela ambiguidade de papéis e pelo conflito de papéis. Os SCG intensificam as informações relevantes para o desenvolvimento do trabalho dos gestores e reduzem a ambiguidade e os conflitos de papéis, assim, contribuem para aumentar o seu desempenho. 
Hall (2008) examinou como os SCG afetam o desempenho gerencial, tendo como premissa que os SCG podem ajudar a melhorar o desempenho gerencial dos empregados da organização, porém, que esse efeito é indireto, mediado por variáveis como o empowerment psicológico e a clareza de papéis no trabalho. A amostra da pesquisa compreendeu 83 gerentes estratégicos de unidades de negócios australianas. Os resultados mostraram que os SCG estão indiretamente relacionados com o desempenho gerencial, pelas variáveis intervenientes empowerment psicológico e clareza de papéis no trabalho. $\mathrm{O}$ estudo de Hall destaca o papel dos mecanismos cognitivos e motivacionais na explicação dos efeitos dos SCG sobre o desempenho gerencial.

Posteriormente, Hall (2011) analisou como a aprendizagem e o desenvolvimento do modelo mental dos gestores ajudam a explicar como os SCG afetam os seus desempenhos individuais. Para isso, investigou uma amostra de 81 gestores de unidades de negócios australianas e observou que SCG mais abrangentes ajudam na aprendizagem e no desenvolvimento dos modelos mentais dos gestores, mas apenas para aqueles que estão há pouco tempo na organização e/ou que trabalham em empresa de pequeno porte. Assim, ele concluiu que os SCG, mediados pela aprendizagem e pelo desenvolvimento do modelo mental, influenciam de forma positiva no desempenho individual dos gestores.

Marginson, McAulay, Roush et al. (2014) investigaram os impactos do empowerment psicológico e da ambiguidade de papéis nas medidas de avaliação de desempenho, especificamente as consequências psicológicas do uso dos sistemas de controle interativo e diagnóstico no desempenho gerencial. Os resultados mostraram que a ambiguidade de papéis é impactada de forma negativa pelo uso diagnóstico e interativo, além de afetar de forma negativa o desempenho gerencial. Denota-se que o uso diagnóstico e o uso interativo são fontes eficazes de informação para neutralizar a ambiguidade de papéis e gerar experiência e autodeterminação psicológica positiva e, indiretamente, melhorar o desempenho.

Com base nos argumentos expostos e nos resultados das pesquisas teórico-empíricas apontadas, formulou-se a terceira hipótese da pesquisa:

- $\mathrm{H}_{3}$ : Há uma relação positiva entre os sistemas de controle gerencial e o desempenho no trabalho, mediada pela criatividade individual.

A confirmação da hipótese $\mathrm{H}_{3}$ indicará que os SCG influenciam de forma positiva diretamente e indiretamente no desempenho individual dos gestores. Dessa forma, os gestores podem se beneficiar dos SCG e da criatividade individual para melhorarem seus desempenhos.

Na Figura 1, apresenta-se o modelo teórico da pesquisa com as hipóteses apontadas.

Figura 1

Modelo teórico da pesquisa

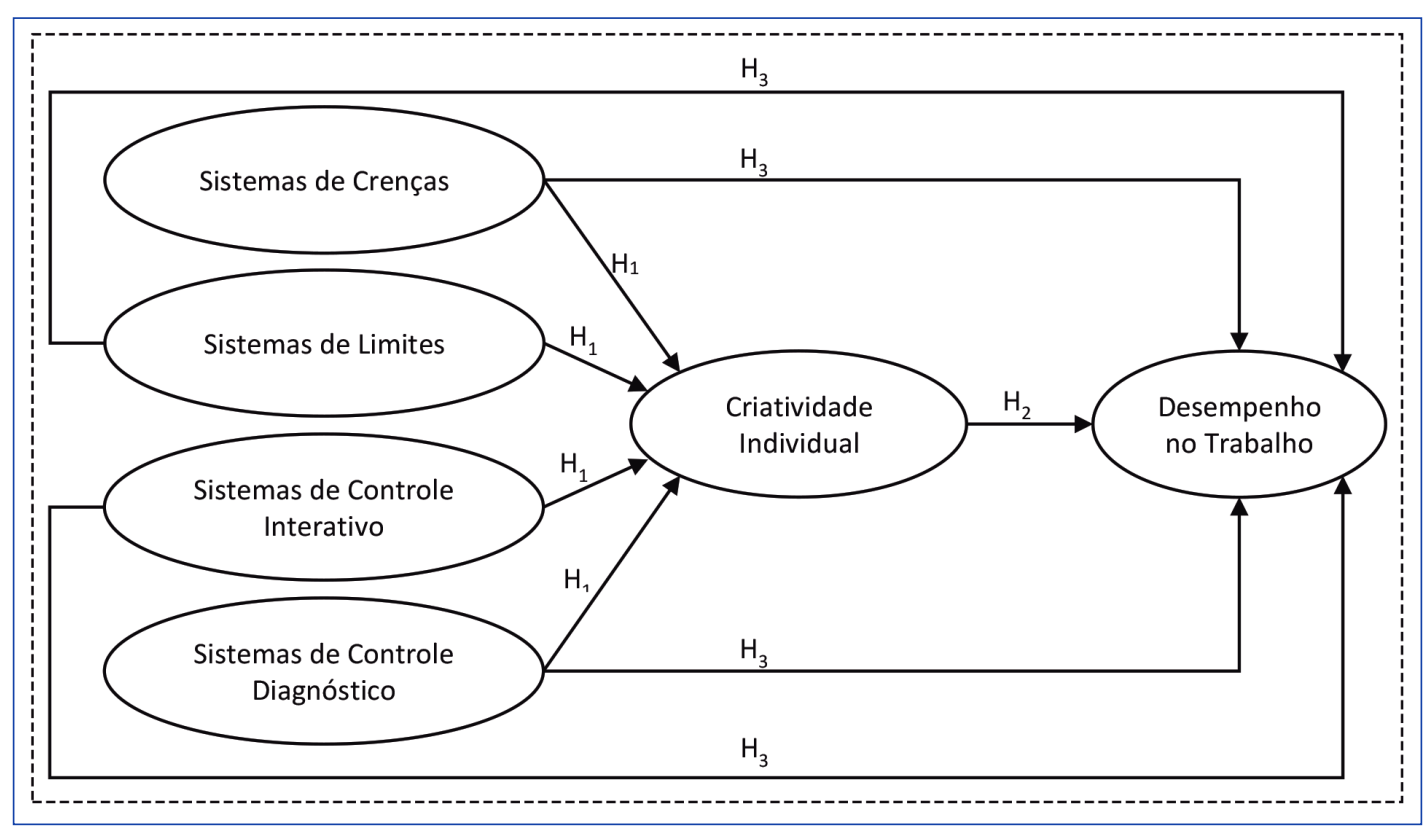

Fonte: Elaborada pelos autores. 
Em conformidade com o modelo teórico da pesquisa, conjectura-se que: os SCG influenciem positivamente a criatividade individual, uma vez que permitem suscitar a criatividade individual nos gestores; a criatividade individual tenha efeito positivo sobre o desempenho no trabalho, o que indicará que os gestores que utilizam novas ideias na prestação de serviços, ou que consideram melhorias nos processos e procedimentos das tarefas melhoram a qualidade do serviço e sua eficácia; e que a criatividade individual seja mediadora dos efeitos dos SCG no desempenho no trabalho.

\section{PROCEDIMENTOS METODOLÓGICOS}

Pesquisa de levantamento foi realizada com as empresas industriais consideradas inovadoras no Brasil, filiadas à Associação Nacional de Pesquisa e Desenvolvimento das Empresas Inovadoras (ANPEI). Um convite foi enviado pelo LinkedIn para os gestores cadastrados no link das empresas inovadoras. No processo de busca, utilizaram-se os termos gerentes, diretores e coordenadores. Dentre 500 convites encaminhados para compor a rede social, 350 foram aceitos pelos gestores, aos quais foi enviado o instrumento de pesquisa pela plataforma SurveyMonkey, no período de fevereiro a junho de 2017, e se obteve um retorno de 126 questionários válidos.

O tamanho da amostra foi calculado pelo software G*Power, o que implica "[...] avaliar o constructo ou variável latente que recebe o maior número de setas ou tem o maior número de preditores" (RINGLE, SILVA e BIDO, 2014, p. 58). O modelo teórico compõe-se de quatro variáveis latentes exógenas (sistemas de controle diagnóstico, sistemas de controle interativo, sistemas de limites e sistemas de crenças) e uma mediadora (criatividade individual). 0 tamanho do efeito utilizado foi de 0,15, o nível de significância de $\alpha$ foi de 0,05 , o poder da amostra de 1-6 foi de 0,8 , com cinco preditores. A amostra mínima exigida para o modelo foi de 92 respondentes.

\section{CONSTRUTOS E INSTRUMENTO DA PESQUISA}

Na Quadro 1, apresentam-se os construtos da pesquisa, as respectivas assertivas e as referências que embasaram ambos.

\section{Quadro 1}

Construto da pesquisa

\begin{tabular}{|c|c|}
\hline Construtos & Assertivas \\
\hline \multirow{4}{*}{$\begin{array}{c}\text { Sistemas de } \\
\text { Crenças } \\
\text { (WIDENER, 2007) }\end{array}$} & $\begin{array}{l}\text { 1. Nossa declaração de missão comunica claramente os valores centrais da empresa para a nossa força } \\
\text { de trabalho. }\end{array}$ \\
\hline & 2. Os gestores de topo comunicam os valores fundamentais da empresa para a nossa força de trabalho. \\
\hline & 3. Nossa força de trabalho está ciente dos valores fundamentais da empresa. \\
\hline & 4. Nossa declaração de missão inspira a nossa força de trabalho. \\
\hline \multirow{4}{*}{$\begin{array}{c}\text { Sistemas de } \\
\text { Limites } \\
\text { (WIDENER, 2007) }\end{array}$} & $\begin{array}{l}\text { 1. Nossa empresa se baseia em um código de conduta empresarial para definir o comportamento } \\
\text { apropriado para a nossa força de trabalho. }\end{array}$ \\
\hline & $\begin{array}{l}\text { 2. O nosso código de conduta empresarial informa a nossa força de trabalho sobre os comportamentos } \\
\text { que estão fora dos limites. }\end{array}$ \\
\hline & 3. Nossa empresa tem um sistema que comunica nossa força de trabalho riscos que devem ser evitados. \\
\hline & 4. Nossa força de trabalho está ciente do nosso código de conduta empresarial. \\
\hline \multirow{4}{*}{$\begin{array}{c}\text { Sistemas de } \\
\text { Controle Interativo } \\
\text { (HENRI, 2006) }\end{array}$} & 1. Promover discussões em reuniões entre superiores, subordinados e pares. \\
\hline & 2. Possibilitar o contínuo desafio e discutir os dados subjacentes, os pressupostos e os planos de ação. \\
\hline & 3. Possibilitar à organização concentrar-se em questões comuns. \\
\hline & 4. Possibilitar à organização focalizar-se nos fatores críticos de sucesso. \\
\hline
\end{tabular}


Continuação

\begin{tabular}{|c|c|}
\hline Construtos & Assertivas \\
\hline \multirow{4}{*}{$\begin{array}{l}\text { Sistemas } \\
\text { de Controle } \\
\text { Diagnóstico } \\
\text { (HENRI, 2006) }\end{array}$} & 1. Acompanhar o progresso em direção às metas. \\
\hline & 2. Monitorar os resultados. \\
\hline & 3. Comparar os resultados com as expectativas. \\
\hline & 4. Revisar as medidas chaves de desempenho. \\
\hline \multirow{8}{*}{$\begin{array}{c}\text { Criatividade } \\
\text { Individual } \\
\text { (MOULANG, 2015) }\end{array}$} & 1. Eu regularmente venho com ideias criativas. \\
\hline & 2. Eu regularmente experimento novos conceitos e ideias. \\
\hline & 3. Eu regularmente realizo tarefas engenhosamente. \\
\hline & 4. Eu frequentemente me envolvo na resolução de problemas de forma inteligente e criativa. \\
\hline & 5. Eu frequentemente busco por inovações e potenciais melhorias dentro da minha unidade de negócio. \\
\hline & $\begin{array}{l}\text { 6. Eu frequentemente crio e avalio várias alternativas para novos problemas dentro de minha unidade } \\
\text { de negócio. }\end{array}$ \\
\hline & 7. Eu frequentemente gero novas perspectivas sobre velhos problemas. \\
\hline & $\begin{array}{l}\text { 8. Eu frequentemente improviso nos métodos de resolução de um problema quando uma resposta não } \\
\text { é aparente. }\end{array}$ \\
\hline \multirow{5}{*}{$\begin{array}{c}\text { Desempenho no } \\
\text { Trabalho } \\
\text { (DENISON, } \\
\text { HOOIJBERG e } \\
\text { QUINN, 1995) }\end{array}$} & 1. Meu desempenho em comparação ao desempenho padrão da empresa. \\
\hline & 2. Meu desempenho em comparação com o desempenho dos meus colegas de trabalho. \\
\hline & 3. Meu desempenho como um modelo exemplar para meus colegas de trabalho. \\
\hline & 4. Meu sucesso gerencial no geral em comparação com o de outros gestores da empresa. \\
\hline & 5. Minha eficácia global enquanto gestor em comparação com a de outros gestores da empresa. \\
\hline
\end{tabular}

Fonte: Elaborado pelos autores.

As assertivas expostas no Quadro 1, após a tradução para a língua portuguesa, foram novamente convertidas para a língua inglesa, a fim de certificar-se sobre sua correta tradução. $O$ instrumento de pesquisa foi inicialmente submetido à avaliação de dois alunos de doutorado em contabilidade e um pesquisador da área, e alguns ajustes de redação foram sugeridos pelos avaliadores. $\mathrm{O}$ questionário foi estruturado em três partes.

Os SCG compõem-se das quatro alavancas de Simons (1995). Para os sistemas de crenças, buscou-se avaliar em que medida as assertivas descrevem os sistemas da organização, sendo 1 = pouco descritivo e 7 = muito descritivo. Para os sistemas de limites, buscou-se avaliar o grau de concordância com as assertivas a respeito deste sistema, sendo 1 = discordo fortemente e 7 = concordo fortemente. Para as assertivas das alavancas de controle interativo e diagnóstico, buscou-se avaliar em que extensão os gestores usam medidas de desempenho, sendo 1 = em pequena extensão e 7 = em grande extensão.

A criatividade individual compõe-se de oito assertivas do questionário de Moulang (2015) que avaliou até que ponto os gestores se envolvem em certas atividades criativas na organização. Nesta questão, buscou-se avaliar em que medida as situações descritas no questionário caracterizam a criatividade do respondente na organização, sendo 1 = quase nunca e 7 = quase sempre.

A mensuração do desempenho no trabalho compõe-se de cinco assertivas do questionário de Denison, Hooijberg e Quinn (1995) que avaliam o desempenho no trabalho do gerente em situações na organização. Trata-se de uma medida de autoavaliação de desempenho e é comumente usada para medir o desempenho individual (WEBSTER, 2006). Este instrumento teve duas adaptações: a primeira, na escala utilizada - no original estava com cinco pontos, neste estudo utilizou-se escala de sete pontos para alinhamento com as demais questões -; e a segunda, na forma de apresentação das assertivas, uma vez que no original havia uma contextualização para cada questão, enquanto que neste optou-se em descrever um enunciado geral. Nessas assertivas, buscou-se avaliar em que medida o desempenho no trabalho dos respondentes coaduna-se com as situações apontadas, sendo 1 = muito abaixo e 7 = muito acima . 


\section{PROCEDIMENTOS DE ANÁLISE DOS DADOS}

Para testar as hipóteses, aplicou-se a Modelagem de Equações Estruturais (Structural Equation Modeling - SEM), valendo-se do software estatístico SmartPLS versão 3. A SEM é uma técnica de teste para modelos teoricamente estimados. Como método de ajuste utilizou-se o de Mínimos Quadrados Parciais (Partial Least Square - PLS), mais indicado para estudos com distribuição de dados não normais, e menos demandante quanto ao tamanho amostral se comparado com a máxima verossimilhança (HAIR JUNIOR, HULT, RINGLE et al., 2014).

O PLS-SEM foi utilizado por ser uma técnica adequada para estudos que possuem amostras pequenas (CHIN, 1998). A análise do PLS-SEM é realizada em duas etapas: a primeira trata da validade de adequação dos construtos, pela relação entre os indicadores e as variáveis latentes ou construtos, e permite que o pesquisador avalie a confiabilidade e a validade das variáveis latentes ou construtos; a segunda trata da relação entre as variáveis latentes ou construtos, e permite verificar se uma variável latente exógena possui relação com uma variável latente endógena (HAIR JUNIOR, HULT, RINGLE et al., 2014).

\section{DESCRIÇÃO E ANÁLISE DOS RESULTADOS}

\section{Modelo de mensuração}

Inicialmente foi testada a validade de adequação dos construtos, tendo sido avaliadas a confiabilidade de consistência interna (confiabilidade composta e alfa de Cronbach), a validade discriminante (cross-loadings e teste de Forell-Larcker) e a validade convergente. A confiabilidade de consistência interna fornece a confiabilidade dos indicadores dentro das variáveis latentes; a validade discriminante é a extensão em que uma variável latente mostra-se distinta das demais variáveis latentes por padrões empíricos; e a validade convergente é a extensão em que um indicador se correlaciona positivamente com indicadores alternativos da mesma variável latente (HAIR JUNIOR, HULT, RINGLE et al., 2014).

O primeiro teste de validade discriminante realizado foi o cross-loadings, no qual as cargas fatoriais das questões devem ser maiores em sua variável latente relacionada do que nas demais (HAIR JUNIOR, HULT, RINGLE et al., 2014). A questão 8 do construto criatividade individual apresentou baixa carga fatorial $(0,427)$, portanto, foi retirada do construto. Desse modo, todas as questões das variáveis latentes apresentaram-se adequadas para o modelo, não sendo necessário retirar mais nenhuma questão, com valores das cargas fatoriais acima de 0,70 (CHIN, 1998).

Após esta análise, realizou-se a avaliação da consistência interna do modelo, pelos testes de Confiabilidade Composta e Alfa de Cronbach, e a validade convergente pelo teste de Average Variance Extracted (AVE). Os resultados são apresentados na Tabela 1.

Tabela 1

Confiabilidade Composta, Alfa de Cronbach, Validade Convergente e Discriminante

\begin{tabular}{|c|c|c|c|c|c|c|c|c|c|}
\hline \multirow[b]{2}{*}{ Variáveis } & \multirow{2}{*}{$\begin{array}{c}\text { Confiabilidade } \\
\text { Composta } \\
>0,70\end{array}$} & \multirow{2}{*}{$\begin{array}{l}\text { Alfa de } \\
\text { Cronbach } \\
>0,70\end{array}$} & \multirow{2}{*}{$\begin{array}{l}\text { AVE } \\
>0,50\end{array}$} & \multicolumn{6}{|c|}{ Validade Discriminante } \\
\hline & & & & $\mathrm{Cl}$ & DT & SCD & $\mathrm{SCl}$ & SCC & $\mathrm{SCL}$ \\
\hline $\begin{array}{l}\text { Criatividade individual } \\
(\mathrm{Cl})\end{array}$ & 0,915 & 0,892 & 0,608 & 0,780 & & & & & \\
\hline $\begin{array}{l}\text { Desempenho no } \\
\text { trabalho (DT) }\end{array}$ & 0,927 & 0,902 & 0,718 & 0,569 & 0,847 & & & & \\
\hline $\begin{array}{l}\text { S. Controle Diagnóstico } \\
\text { (SCD) }\end{array}$ & 0,933 & 0,905 & 0,777 & 0,323 & 0,203 & 0,882 & & & \\
\hline $\begin{array}{l}\text { S. Controle Interativo } \\
(\mathrm{SCl})\end{array}$ & 0,937 & 0,912 & 0,789 & 0,206 & 0,011 & 0,559 & 0,888 & & \\
\hline $\begin{array}{l}\text { Sistemas de Crenças } \\
\text { (SCC) }\end{array}$ & 0,940 & 0,914 & 0,795 & 0,354 & 0,106 & 0,635 & 0,786 & 0,892 & \\
\hline $\begin{array}{l}\text { Sistemas de Limites } \\
(\mathrm{SCL})\end{array}$ & 0,936 & 0,914 & 0,784 & 0,128 & $-0,021$ & 0,618 & 0,500 & 0,496 & 0,886 \\
\hline
\end{tabular}

Fonte: Dados da pesquisa. 
De acordo com a Tabela 1, todas as cargas para a AVE mostram-se estatisticamente significativas, uma vez que os valores de cada variável latente são superiores ao valor limite de 0,50 . Portanto, as variáveis latentes explicam mais da metade da variância dos seus indicadores (HAIR JUNIOR, BLACK, BABIN et al., 2009). Quanto aos resultados dos coeficientes de Confiabilidade Composta e do Alfa de Cronbach, que têm a finalidade de testar a confiabilidade do modelo, verifica-se que os coeficientes de ambos apresentam-se superiores ao valor mínimo 0,70, conforme recomendado por Hair Junior, Black, Babin et al. (2009).

Nota-se validade discriminante entre os construtos, todos os indicadores apresentaram cargas fatoriais maiores em suas respectivas variáveis latentes (HAIR JUNIOR, BLACK, BABIN et al., 2009), por exemplo, a criatividade individual $(0,780)$. Não há correlação entre as variáveis latentes de primeira ordem, que excedem em magnitude a raiz quadrada da AVE das variáveis latentes relacionadas (HAIR JUNIOR, BLACK, BABIN et al., 2009). Por exemplo, o maior valor da criatividade individual ocorreu com o desempenho no trabalho $(0,569)$, mas foi menor que as suas respectivas AVE (criatividade individual $=0,608$ e desempenho no trabalho $=0,718$ ).

\section{Modelo estrutural}

Após a verificação da adequação do modelo estrutural, realizaram-se as estimativas das equações estruturais por meio da análise do bootstrapping, técnica adotada para testar a significância das relações entre as variáveis latentes utilizadas no estudo (HAIR JUNIOR, HULT, RINGLE et al., 2014). A Tabela 2 mostra as relações dos coeficientes estimados do modelo estrutural (efeito direto, indireto e total), os níveis de significância, a Variance Accounted For (VAF) e o resultado do teste das hipóteses da pesquisa.

Tabela 2

Resultados do modelo estrutural

\begin{tabular}{|c|c|c|c|c|c|c|c|c|c|c|c|}
\hline \multirow{2}{*}{ Descrição } & \multicolumn{2}{|c|}{$\begin{array}{l}\text { Modelo sem } \\
\text { mediação }\end{array}$} & \multicolumn{9}{|c|}{ Modelo com mediação } \\
\hline & $\begin{array}{l}\text { Coef. } \\
\text { Estr. }\end{array}$ & $\begin{array}{c}\text { Valor } \\
p\end{array}$ & $\begin{array}{l}\text { Efeito } \\
\text { Direto }\end{array}$ & $\begin{array}{c}\text { Valor } \\
\mathrm{p}\end{array}$ & $\begin{array}{l}\text { Efeito } \\
\text { Indireto }\end{array}$ & $\begin{array}{c}\text { Valor } \\
\mathrm{p}\end{array}$ & $\begin{array}{l}\text { Efeito } \\
\text { Total }\end{array}$ & $\begin{array}{c}\text { Valor } \\
\mathrm{p}\end{array}$ & $\begin{array}{l}\text { Mediação } \\
\text { (VAF) }\end{array}$ & \multicolumn{2}{|r|}{ Hipótese } \\
\hline $\begin{array}{l}\text { S.Controle Diagnóstico } \rightarrow \\
\text { Criatividade Individual }\end{array}$ & - & - & $-0,190$ & 0,088 & - & - & $-0,190$ & 0,088 & - & $\mathrm{H}_{1}$ & Rejeita-se \\
\hline $\begin{array}{l}\text { S.Controle Diagnóstico } \rightarrow \\
\text { Desempenho no Trabalho }\end{array}$ & 0,002 & 0,989 & $-0,074$ & 0,405 & $-0,108$ & 0,092 & $-0,182$ & 0,127 & - & $\mathrm{H}_{3}$ & Rejeita-se \\
\hline $\begin{array}{l}\text { S.Controle Interativo } \rightarrow \\
\text { Criatividade Individual }\end{array}$ & - & - & 0,411 & 0,002 & - & - & 0,411 & 0,002 & - & $\mathrm{H}_{1}$ & Aceita-se \\
\hline $\begin{array}{l}\text { S.Controle Interativo } \rightarrow \\
\text { Desempenho no Trabalho }\end{array}$ & 0,000 & 0,999 & $-0,105$ & 0,393 & 0,234 & 0,004 & 0,129 & 0,247 & - & $\mathrm{H}_{3}$ & Rejeita-se \\
\hline $\begin{array}{l}\text { Sistemas de Crenças } \rightarrow \\
\text { Criatividade Individual }\end{array}$ & - & - & 0,253 & 0,010 & - & - & 0,253 & 0,010 & - & $\mathrm{H}_{1}$ & Aceita-se \\
\hline $\begin{array}{l}\text { Sistemas de Crenças } \rightarrow \\
\text { Desempenho no Trabalho }\end{array}$ & 0,382 & 0,008 & 0,210 & 0,062 & 0,144 & 0,010 & 0,355 & 0,006 & 0,289 & $\mathrm{H}_{3}$ & $\begin{array}{l}\text { Aceita-se } \\
\text { parcialmente }\end{array}$ \\
\hline $\begin{array}{l}\text { Sistemas de Limites } \rightarrow \\
\text { Criatividade Individual }\end{array}$ & - & - & $-0,137$ & 0,206 & - & - & $-0,137$ & 0,206 & - & $\mathrm{H}_{1}$ & Rejeita-se \\
\hline $\begin{array}{l}\text { Sistemas de Limites } \rightarrow \\
\text { Desempenho no Trabalho }\end{array}$ & $-0,277$ & 0,023 & $-0,135$ & 0,073 & $-0,078$ & 0,228 & $-0,214$ & 0,031 & - & $\mathrm{H}_{3}$ & Rejeita-se \\
\hline $\begin{array}{l}\text { Criatividade Individual } \rightarrow \\
\text { Desempenho no Trabalho }\end{array}$ & - & - & 0,571 & 0,000 & - & - & 571 & 0,000 & - & $\mathrm{H}_{2}$ & Aceita-se \\
\hline$R^{2}$ & \multicolumn{2}{|c|}{ DT: 0,093 } & \multicolumn{9}{|c|}{ Cl: 0,170 e DT: 0,358} \\
\hline
\end{tabular}

Fonte: Dados da pesquisa.

Pelos coeficientes de determinação $\left(R^{2}\right)$, a criatividade individual é explicada em $17,0 \%$ e o desempenho no trabalho em $35,8 \%$ pelo modelo com mediação. Em estudos anteriores, observam-se valores baixos para os $R^{2}$ da criatividade e desempenho no trabalho quando relacionado com os SCG. Para a variável criatividade, o maior $R^{2}$ encontrado foi no estudo de Moulang 
(2015), com 0,226. Para a variável desempenho, o maior $R^{2}$ encontrado foi no estudo de Hall (2008), com 0,529. Desse modo, o $R^{2}$ deste estudo é considerado válido, visto que os valores foram próximos dos valores encontrados pelos estudos anteriores.

Nos resultados das relações, o efeito direto representa a relação direta entre a variável exógena (desempenho no trabalho), mediadora (criatividade individual) e endógena (desempenho no trabalho) no modelo. $O$ efeito indireto representa a relação entre as variáveis exógena (SCG) e endógena (desempenho no trabalho) por meio da mediadora (criatividade individual) na construção do modelo de equações estruturais. O efeito total é a soma do efeito direto e do efeito indireto entre as variáveis exógena (SCG) e endógena (desempenho no trabalho) no modelo de equações estruturais PLS (HAIR JUNIOR, BLACK, BABIN et al., 2009).

Nota-se que os sistemas de controle interativo e de crenças têm efeito direto positivo sobre a criatividade individual, enquanto os sistemas de controle diagnóstico têm efeito negativo. Apenas os sistemas de crenças apresentaram efeito direto positivo sobre o desempenho no trabalho. Já os sistemas de limites apresentaram efeito direto negativo sobre o desempenho no trabalho. Finalmente, a criatividade individual também apresentou efeito direto positivo sobre o desempenho no trabalho.

Para os efeitos indiretos, os sistemas de controle diagnóstico, interativo e de crenças possuem efeito indireto sobre o desempenho no trabalho pela mediação da criatividade individual. Contudo, se a relação direta entre as variáveis exógena e endógena não for significativa, não há sentido em analisar o efeito indireto pela mediação (HAIR JUNIOR, BLACK, BABIN et al., 2009). Pelo efeito total, pode-se afirmar que os sistemas de controle interativo e de crenças estão positivamente relacionados com criatividade individual, enquanto os sistemas de controle diagnóstico estão negativamente relacionados. Outrossim, os sistemas de crenças e a criatividade individual estão positivamente relacionados com o desempenho, enquanto os sistemas de limites estão negativamente relacionados com o desempenho no trabalho.

\section{ANÁLISE DAS HIPÓTESES E DISCUSSÃO DOS RESULTADOS}

A hipótese $\mathrm{H}_{1}$ prevê relação positiva entre os SCG e a criatividade individual, assim, diante dos resultados, aceita-se a $\mathrm{H}_{1}$ para os sistemas de controle interativo e de crenças. Os sistemas de controle interativo e de crenças influenciam positivamente na criatividade individual dos gestores. Estes resultados corroboram a pesquisa de Moulang (2015), que analisou o uso interativo do SCG e constatou relação positiva com a criatividade individual; e a de Speklé, Van Elten e Widener (2017), que analisou as quatro alavancas em conjunto.

Simons (1995) propôs que as organizações precisam de novos e diferentes tipos de sistemas de controle para capacitar os funcionários e facilitar o pensamento criativo e as atividades inovadoras. Nesse sentido, Speklé, Van Elten e Widener (2017) observaram que quanto mais intensamente as alavancas dos SCG forem utilizadas, mais claro se torna o controle nas organizações, o que fornece motivação adicional para os funcionários serem criativos em suas tarefas. Moulang (2015) constatou que os SCG possuem o potencial de encorajar a criatividade nos indivíduos, fornecendo informações úteis de feedback e alinhando o comportamento dos indivíduos com os objetivos organizacionais.

Positivamente, os sistemas de crenças capacitam e motivam os gestores a terem iniciativas na busca de oportunidades, na tomada de decisões e na solução de problemas de forma condizente com os valores da organização, facilitando o comportamento criativo (SIMONS, 1995). A relação positiva e significativa encontrada neste estudo, entre os sistemas de crenças e a criatividade individual, com um coeficiente de caminho de 0,253, suporta o preconizado na literatura.

Os sistemas de controle interativo podem estimular a criatividade e a inovação de produtos, oferecer orientação, desencadear iniciativas e proporcionar legitimidade (BISBE e OTLEY, 2004). Portanto a relação positiva e significativa encontrada neste estudo entre os sistemas de controle interativo e a criatividade individual, com um coeficiente de caminho de 0,396, corrobora os resultados de pesquisas veiculadas pela literatura (BISBE e OTLEY, 2004), de que os sistemas de controle interativo estimulam os gestores a buscar oportunidades estratégicas e criativas.

No entanto, os sistemas de controle diagnóstico e de limites apresentaram relação negativa significativa e não significativa, respectivamente, assim rejeita-se a $\mathrm{H}_{1}$ para essas variáveis. Os sistemas de controle diagnóstico são usados para motivar, monitorar e premiar o alcance das metas, já os sistemas de limites são usados para estabelecer limites aos participantes da organização (SIMONS, 1995). Ambos atuam como fatores de restrição do comportamento dos funcionários e representam uma 
força negativa (SIMONS, 1995). Assim, o sinal negativo da relação desses sistemas com a criatividade individual é justificado por restringirem a criatividade dos gestores em suas atividades.

Já a hipótese $\mathrm{H}_{2}$ prevê relação positiva entre a criatividade individual e o desempenho no trabalho. Os resultados revelam, com nível de significância de $1 \%$, que a criatividade individual está positivamente relacionada com o desempenho no trabalho, com um coeficiente de caminho de 0,567 . Isso indica que gestores criativos, por meio de novas ideias, conseguem potencializar as suas atividades, diminuindo as dificuldades para efetivar suas tarefas. Os resultados confirmam a $\mathrm{H}_{2}$ e corroboram os estudos de Gong, Huang e Farh (2009), Zhang e Bartol (2010) e Bodla e Naeem (2014).

Gong, Huang e Farh (2009), pela regressão hierárquica, encontraram relação positiva e significativa entre a criatividade do empregado e o desempenho no trabalho, indicando que indivíduos criativos podem aperfeiçoar procedimentos ou processos para aumentar sua eficiência. Zhang e Bartol (2010), pela regressão não linear, encontraram relação positiva e significativa entre desempenho criativo e desempenho global no trabalho, indicando que, para que tenham um melhor desempenho em tarefas complexas, os gestores precisam incorporar ideias novas. Bodla e Naeem (2014), pela modelagem de equações estruturais, encontraram relação positiva e significativa de efeito direto entre o desempenho criativo e o volume de vendas individuais, indicando que vendedores criativos são hábeis para captar a necessidade do cliente.

Finalmente, a hipótese $\mathrm{H}_{3}$ postula que há relação positiva entre os SCG e o desempenho no trabalho, mediada pela criatividade individual. Os resultados sugerem que os sistemas de crenças estão positivamente relacionados com o desempenho no trabalho, com nível de significância de 1\%, e que a criatividade individual tem efeito parcial entre as duas variáveis, já que o VAF foi de 0,289. Segundo Hair Junior, Black, Babin et al. (2009), com um VAF acima de 0,8 a mediação é completa, entre 0,2 e 0,8 a mediação é parcial e com valores menores que 0,2 não existe mediação. Assim, a $\mathrm{H}_{3}$ foi confirmada parcialmente para os sistemas de crenças, visto que a mediação foi parcial. Este resultado difere do estudo de Webster (2006), que não encontrou relação entre uso interativo dos SCG e o desempenho no trabalho, mediado pela criatividade, o que instiga pesquisas adicionais.

Para os sistemas de limites, a relação foi negativa e significativa com o desempenho do trabalho, logo, rejeita-se a hipótese $\mathrm{H}_{3}$ para esta variável. Os sistemas de limites estabelecem as regras do jogo e identificam ações e armadilhas que os funcionários devem evitar, ou seja, atuam como uma restrição às ações dos gestores (SIMONS, 1995). Essas restrições podem prejudicar o desempenho dos gestores pois, ao ter a execução de suas atividades limitada de determinada forma, podem tomar decisões que não gostariam, prejudicando o desempenho no trabalho.

A não significância da relação dos sistemas de controle diagnóstico e interativo com o desempenho no trabalho pode indicar duas suposições. Primeiramente, nos sistemas de controle diagnóstico, os gestores exigem da empresa comunicação clara sobre o modo de executar seu trabalho, para que possam ajustar sua rotina e obter benefícios com o uso dos SCG. Já em relação aos sistemas de controle interativo, eles têm discernimento de que a execução de suas tarefas pode ser ajustada ou reorganizada conforme as necessidades para melhor cumprir os objetivos. Desse modo, a relação não significante pode ser justificada pelo equilíbrio de ambas as alavancas de controle (diagnóstico/interativo) nas empresas pesquisadas.

Destaca-se que os sistemas de controle interativo e de crenças influenciam na criatividade individual, tal como a criatividade influencia o desempenho no trabalho, além de ser uma mediadora parcial entre os sistemas de crenças e o desempenho do trabalho. Isso sugere que os sistemas de controle interativo estimulam os gestores a utilizarem a criatividade para conduzir suas atividades, e os de crenças os capacitam e motivam para resolverem os problemas existentes na organização, promovendo a criatividade (SIMONS, 1995). Os sistemas de crenças melhoram o desempenho dos gestores, auxiliando assim na solução de problemas da organização (SIMONS, 1995), e a criatividade auxilia de forma parcial na relação entre ambos, uma vez que ela diminui as dificuldades para efetivar as tarefas na organização (GONG, HUANG e FARH, 2009). 


\section{CONCLUSÕES}

Este estudo analisou a influência dos sistemas de controle gerencial, mediada pela criatividade individual, sobre o desempenho no trabalho. Os resultados da pesquisa fornecem suporte para as proposições de Simons (1995) quanto à relevância dos sistemas de controle interativo e de crenças na geração de criatividade individual dos gestores. Estes dois sistemas são necessários para capacitar os gestores e permitir que a criatividade floresça em suas ações, auxiliando na condução das tarefas na organização, o que suporta a hipótese $\mathrm{H}_{1}$ e corrobora os estudos de Moulang (2015) e Speklé, Van Elten e Widener (2017).

Os resultados sinalizam que a criatividade individual influencia de forma positiva o desempenho no trabalho. Gestores criativos conseguem potencializar suas atividades e melhorar o desempenho no trabalho, o que ressalta a importância da geração de ideias criativas pelos gestores. Os resultados confirmam, portanto, a hipótese $\mathrm{H}_{2}$ e se coadunam com os resultados das pesquisas de Gong, Huang e Farh (2009), Zhang e Bartol (2010) e Bodla e Naeem (2014).

Observa-se ainda que os sistemas de crenças influenciam de forma positiva o desempenho no trabalho, e que a criatividade individual auxilia de forma parcial na relação entre ambos. Os sistemas de crenças são usados pelas organizações para inspirar a busca por novas oportunidades, incentivando os gestores a darem o melhor de si em suas tarefas na organização, melhorando o seu desempenho. Além disto, eles fomentam a criatividade individual, a qual, de forma parcial, medeia ambos, aceitando-se, assim, parcialmente, $\mathrm{a}_{3}$.

Conclui-se com base nos resultados que os SCG nas organizações pesquisadas são influenciadores da criatividade individual e do desempenho no trabalho. Destaca-se, especificamente, que os sistemas de controle interativo e de crenças influenciam na criatividade individual, tal como a criatividade influencia o desempenho no trabalho, além de ser uma mediadora parcial entre os sistemas de crenças e o desempenho do trabalho.

O estudo contribui para a literatura ao abordar em conjunto as alavancas de Simons, a criatividade individual e o desempenho no trabalho em empresas industriais consideradas inovadoras no Brasil, embora estudos anteriores já tenham analisado a relação das alavancas de Simons com a criatividade individual (MOULANG, 2015; SPEKLÉ, VAN ELTEN e WIDENER, 2017). Com relação à prática no cotidiano das empresas, este estudo também vem a contribuir pois ele evidencia o valor da criatividade individual no desempenho dos gestores, principalmente pela mediação parcial da relação entre os sistemas de crenças e o desempenho no trabalho.

Dentre as limitações, destaca-se que este estudo focalizou o efeito positivo da criatividade individual sobre o desempenho dos gestores, mas é possível que a criatividade tenha efeito negativo, conduzindo os gestores a se engajarem em comportamentos desonestos (GINO e ARIELY, 2012). Outra limitação está nos instrumentos de pesquisa, embora tenham sido extraídas de estudos anteriores, as assertivas podem não ter captado completamente a natureza dos SCG, da criatividade individual e do desempenho no trabalho.

Como possível desdobramento do presente estudo, recomendam-se pesquisas longitudinais que possam fornecer evidências empíricas da causalidade nas relações entre os construtos analisados. Além disso, outras variáveis de mediação podem ser consideradas, como o comportamento do indivíduo, a satisfação no trabalho ou a autonomia do papel. Recomenda-se ainda que pesquisas futuras segreguem os tipos de estrutura das organizações entre aquelas que são mais e menos flexíveis, podendo ser entendidas como mais ou menos burocráticas, para averiguar eventuais diferenças. 


\section{REFERÊNCIAS}

ADLER, P. S.; CHEN, C. X. Combining creativity and control: Understanding individual motivation in large-scale collaborative creativity. Accounting, Organizations and Society, v. 36, n. 2, p. 63-85, 2011.

AMABILE, T. M. The social psychology of creativity: A componential conceptualization. Journal of Personality and Social Psychology, v. 45 , n. 2, p. 357, 1983.

ANTHONY, R. Planning and control systems: a framework for analysis. Division of Research, Graduate School of Business Administration, Harvard University. Boston: Harvard University, 1965.

BISBE, J.; OTLEY, D. The effects of the interactive use of management control systems on product innovation. Accounting, Organizations and Society, v. 29, n. 8, p. 709-737, 2004.

BODLA, M. A.; NAEEM, B. Creativity as mediator for intrinsic motivation and sales performance. Creativity Research Journal, v. 26, n. 4, p. 468-473, 2014.

BURNEY, L.; WIDENER, S. K. Strategic performance measurement systems, job-relevant information, and managerial behavioral responses - role stress and performance. Behavioral Research in Accounting, v. 19, n. 1, p. 43-69, 2007.

CHIN, W. W. The partial least squares approach for structural equation modelling. In: MARCOULIDES, G. A. (Ed.). Modern methods for business research. New York: Lawrence Erlbaum, 1998. p. 295-336.

DAVILA, A.; FOSTER, G.; OYON, D. Accounting and control, entrepreneurship and innovation: Venturing into new research opportunities. European Accounting Review, v. 18, n. 2, p. $281-$ 311, 2009.

DENISON, D. R.; HOOIJBERG, R.; QUINN, R. E. Paradox and performance: Toward a theory of behavioral complexity in managerial leadership. Organization Science, v. 6, n. 5, p. 524-540, 1995.

GEORGE, J. M.; ZHOU, J. Understanding when bad moods foster creativity and good ones don't: the role of context and clarity of feelings. Journal of Applied Psychology, v. 87, n. 4, p. 687, 2002.

GILSON, L. L. Why be creative: A review of the practical outcomes associated with creativity at the individual, group, and organizational levels. In: ZHOU, J.; SHALLEY, C. (Eds.). Handbook of Organizational Creativity. New York: Lawrence Erlbaum, 2008. p. 303-322.

GINO, F.; ARIELY, D. The dark side of creativity: original thinkers can be more dishonest. Journal of Personality and Social Psychology, v. 102, n. 3, p. 445, 2012.

GONG, Y.; HUANG, J.-C.; FARH, J.-L. Employee learning orientation, transformational leadership, and employee creativity: The mediating role of employee creative self-efficacy. Academy of Management Journal, v. 52, n. 4, p. 765-778, 2009.

HAIR JUNIOR, J. F. et al. Análise multivariada de dados. Porto Alegre: Bookman, 2009.
HAIR JUNIOR, J. F. et al. Primer on Partial Least Squares Structural Equation Modeling (PLS-SEM). Los Angeles: Sage, 2014.

HALL, M. The effect of comprehensive performance measurement systems on role clarity, psychological empowerment and managerial performance. Accounting, Organizations and Society, v. 33, n. 2-3, p. $141-163,2008$.

HALL, M. Do comprehensive performance measurement systems help or hinder managers' mental model development? Management Accounting Research, v. 22, n. 2, p. 68-83, 2011.

HENRI, J.-F. Management control systems and strategy: A resourcebased perspective. Accounting, Organizations and Society, v. 31, n. 6, p. 529-558, 2006.

MARGINSON, D. et al. Examining a positive psychological role for performance measures. Management Accounting Research, v. 25, n. 1, p. 63-75, 2014.

MERCHANT, K. A.; VAN DER STEDE, W. A. Management control systems: performance measurement, evaluation and incentives. 4. ed. New York: Pearson, 2017

MOULANG, C. Performance measurement system use in generating psychological empowerment and individual creativity. Accounting \& Finance, v. 55, n. 2, p. 519-544, 2015.

RINGLE, C. M.; SILVA, D.; BIDO, D. Z. Modelagem de equações estruturais com utilização do SmartPLS. Revista Brasileira de Marketing, v. 13, n. 2, p. 56-73, 2014.

SIMONS, R. Levers of control: how managers use innovative control systems to drive strategic renewal. Boston: Harvard Business School, 1995.

SPEKLÉ, R. F.; VAN ELTEN, H. J.; WIDENER, S. K. Creativity and control: A paradox-Evidence from the levers of control framework. Behavioral Research in Accounting, v. 29, n. 2, p. 73-96, 2017.

WEBSTER, C. An empirical analysis of the relationships between the interactive use of performance measurement systems, creativity and performance: the intervening role of psychological empowerment. In: ANNUAL CONGRESS OF THE EUROPEAN ACCOUNTING, 29., 2006, Dublin. Proceedings... Brussels: European Accounting Association, 2006.

WIDENER, S. K. An empirical analysis of the levers of control framework. Accounting, Organizations and Society, v. 32, n. 7-8, p. 757-788, 2007.

ZHANG, X.; BARTOL, K. M. The influence of creative process engagement on employee creative performance and overall job performance: $A$ curvilinear assessment. Journal of Applied Psychology, v. 95, n. 5, p. 862, 2010.

ZHOU, J.; SHALLEY, C. E. Expanding the scope and impact of organizational creativity research. In: ZHOU, J.; SHALLEY, C. (Eds.). Handbook of Organizational Creativity. New York: Lawrence Erlbaum, 2008. p. 347-368. 
Itzhak David Simão Kaveski

ORCID: https://orcid.org/0000-0001-8691-9904

Doutor em Contabilidade pela Universidade Federal de Santa Catarina (UFSC); Professor da Universidade Federal de Mato Grosso do Sul (UFMS), Corumbá - MS, Brasil. E-mail: itzhak.konoha@gmail.com

\section{Ilse Maria Beuren}

ORCID: https://orcid.org/0000-0003-4007-6408

Doutora em Controladoria e Contabilidade pela Universidade de São Paulo (FEA/USP); Professora do Programa de Pós-Graduação em Contabilidade da Universidade Federal de Santa Catarina (UFSC), Florianópolis - SC, Brasil. E-mail: ilse.beuren@gmail.com 